\title{
Hermite cubic spline collocation methods with upwind features
}

\author{
Weiwei Sun*
}

(Received 7 August 2000)

\begin{abstract}
In this paper, we present an eigenvalue analysis of the first-order Hermite cubic spline collocation differentiation matrices with arbitrary collocation points. Some important features are explored and the method is compared with some other discrete methods, such as finite difference methods. A class of spline collocation methods with upwind features is proposed for solving singular perturbation problems.
\end{abstract}

*Department of Mathematics, City University of Hong Kong, Kowloon, Hong Kong. mailto:maweiw@maths.cityu.edu.hk

${ }^{0}$ See http://anziamj.austms.org.au/V42/CTAC99/Sun for this article and ancillary services, (C) Austral. Mathematical Soc. 2000. Published 27 Nov 2000. 


\section{Contents}

1 Introduction $\quad$ C1380

2 Hermite cubic collocation methods $\quad$ C1383

3 Stable collocation points

C1384

4 Numerical examples

C1391

4.1 Example 1.................... . . C1391

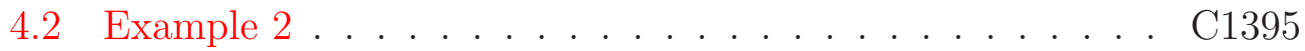

References

C1395

\section{Introduction}

The spline collocation method has been extensively applied in numerical PDEs and oDEs $[2,3]$ due to its ease of implementation and high-order accuracy. Collocation at Gauss points produces a high-order accuracy for many physical problems. However, for those convection-dominated flows and perturbation problems, collocation at Gauss points can produce bad oscillations.

A well-known technique for such problems is an upwinding scheme which can be well-posed for finite difference and finite element methods. Collocation at Gauss points produces many features of central finite differences [7] which 
is known not to be efficient for such problems. Collocation with upwinding feature is of high interest.

Collocation methods with upwinding features were proposed by Allan and Pinder [1] for a convection-dispersion transport equation. In their approach, Hermite cubic spline collocation with some nonsymmetric collocation points are used and this is shown to produce oscillation-free solutions to the linear problem. Ringhofer [6] considered a class of quasilinear singular perturbed boundary value problems by using spline collocation with some specified nonsymmetric collocation points. The stability of his method was analysed. Mahmood and Osborne proposed a noncompact scheme for the reduced equation of such singular perturbation problems. The scheme is stable when some nonsymmetric collocation points are applied. Some upwinding features for pseudospectral collocation methods were studied by Huang and Sloan [4]. Numerical results suggest that the eigenvalues of the first-order differentiation matrix are located in the left-half plane. This is the main feature of upwinding scheme.

A simple example to be considered here is

$$
\epsilon u_{x x}-u_{x}+d(x)=0
$$

subject to appropriate boundary conditions. This model problem has been considered in those previous works and has its roots in many physical problems. Ringhofer [6] and Huang and Sloan [5] considered the spatial problem and Allan and Pinder [1] examined the corresponding time-dependent prob- 
lem. A numerical method applied to (1) gives the discrete form

$$
\epsilon T_{2} u-p T_{1} u+d=0
$$

where $T_{1}$ and $T_{2}$ are the first-order and the second-order differentiation matrices of the method used. A linear stability analysis shows that the stability depends upon the eigenvalues of the matrix

$$
M=\epsilon T_{2}-p T_{1}
$$

For $\epsilon$ sufficiently small, the scheme is stable when all the eigenvalues of $T_{1}$ are located in the left-half plane. This requirement is satisfied by the classical upwinding finite difference methods and upwinding finite element methods.

In this paper, we consider Hermite cubic spline collocation methods which possess such upwinding features. Numerical investigation of the stable collocation point set in which all eigenvalues of the first-order differentiation matrix are located in the left-half plane was presented in [8]. However, no theoretical analysis for the stable collocation point set has been presented. The primary purpose of this paper is to present an eigenvalue analysis of the first-order Hermite cubic spline collocation differentiation matrix. Based on this analysis, we present the collocation point set in which the Hermite cubic spline collocation method is stable. These stable collocation methods are applied for solving some simple perturbed problem and compared with classical collocation methods based on Gauss points. 


\section{Hermite cubic collocation methods}

First, we describe briefly the spline collocation formulation. Let $\Pi_{N}=$ $\left\{x_{i}\right\}_{i=1}^{N+1}$ be a uniform mesh on $[0,1]$ so that $h=x_{i+1}-x_{i}, i=1, \ldots, N$. The Hermite cubic approximation $v(x)$ is defined by

$$
v(x):=\xi_{1}(s) u_{i}+h \xi_{2}(s) u_{i}^{\prime}+\xi_{3}(s) u_{i+1}+h \xi_{4}(s) u_{i+1}^{\prime} \quad s \in[0,1]
$$

on each element $\left[x_{i}, x_{i+1}\right](i=1,2, \ldots, N)$. Here, $\xi_{i}(s), i=1,2,3,4$, are the standard shape functions. Let

$$
\Omega=\{(x, y) \mid x<y ; x, y \in(0,1)\} \in \mathbb{R}^{2}
$$

and we denote the collocation points by $\Pi_{C O L}:=\left\{x_{i j}^{c}\right\}_{i, j=1}^{N, 2}$, where

$$
\begin{array}{ll}
x_{i 1}^{c}=x_{i}+\sigma_{1}\left(x_{i+1}-x_{i}\right), & i=1,2, \ldots, N, \\
x_{i 2}^{c}=x_{i}+\sigma_{2}\left(x_{i+1}-x_{i}\right), & i=1,2, \ldots, N
\end{array}
$$

and $\left(\sigma_{1}, \sigma_{2}\right) \in \Omega$.

We apply the spline collocation method for solving (1) with Dirichlet boundary conditions. This spline collocation discrete system is given by

$$
\epsilon v^{\prime \prime}\left(x_{i l}^{c}\right)-p v^{\prime}\left(x_{i l}^{c}\right)=-d\left(x_{i l}^{c}\right), \quad i=1,2, \ldots, N ; l=1,2 .
$$

In matrix form, we have

$$
\epsilon A_{2} \bar{u}-p A_{1} \bar{u}=d
$$


where $A_{k}$ denotes the $k$ th-order collocation differentiation matrix, $k=0,1,2$, which is the approximation to the $k$ th-order differential operator $u^{(k)}, k=$ $0,1,2$ and

$$
\left(A_{k} v\right)_{l}=v^{(k)}\left(x_{i j}^{c}\right), \quad k=0,1,2 ; j=1,2 ; i=1,2, \ldots, N .
$$

The relation between $l$ and $i, j$ and the corresponding structure of $A_{k}$ depends upon the ordering of the collocation points and the unknowns for $v(x)$. For the natural ordering $l=2(i-1)+j$, we set

$$
\begin{aligned}
& \bar{u}=\left[u_{1}, h u_{1}^{\prime}, u_{2}, h u_{2}^{\prime}, \ldots, u_{N}, h u_{N}^{\prime}\right]^{T}, \\
& \Pi_{C O L}=\left\{x_{11}^{c}, x_{12}^{c}, x_{21}^{c}, x_{22}^{c}, \ldots, x_{N 1}^{c}, x_{N 2}^{c}\right\} .
\end{aligned}
$$

Let $v=\left(v\left(x_{11}^{c}\right), v\left(x_{12}^{c}\right), v\left(x_{21}^{c}\right), \ldots, v\left(x_{N 2}^{c}\right)\right)$. Then $v=A_{0} \bar{u}$ and

$$
\epsilon T_{2} v-p T_{1} v=d
$$

where $T_{1}=A_{1} A_{0}^{-1}$ and $T_{2}=A_{2} A_{0}^{-1}$.

\section{Stable collocation points}

We consider the following eigenvalue problem

$$
A_{1} v=\lambda A_{0} v
$$


where $A_{k}$ denotes the $k$-order Hermite spline collocation differentiation matrix with respect to Dirichlet boundary conditions. Theoretical analysis for the eigenvalue problem with symmetric collocation points and numerical investigation for nonsymmetric collocation points were studied in [8]. Let $\lambda\left(A_{1}^{-1} A_{0}\right)$ denote the eigenvalues of $A_{1}^{-1} A_{0}$. Given $\Pi_{N}$ these are dependent upon the choice of collocation point $\left(\sigma_{1}, \sigma_{2}\right)$. The stable collocation point sets are defined by

$$
\begin{aligned}
& S_{1}^{D^{+}}=\left\{\left(\sigma_{1}, \sigma_{2}\right) \mid \Re\left(\lambda\left(A_{1} A_{0}^{-1}\right)\right)>0\right\} \\
& S_{1}^{D^{-}}=\left\{\left(\sigma_{1}, \sigma_{2}\right) \mid \Re\left(\lambda\left(A_{1} A_{0}^{-1}\right)\right)<0\right\} .
\end{aligned}
$$

Some properties of the first-order spline collocation differentiation matrix are given in [8]. These have been obtained by using a so-called local condensation technique $[2,5,9,10]$.

Lemma $1[8]$

$$
\operatorname{det}\left(A_{1}-\lambda A_{0}\right)=h^{-2 N} \operatorname{det}\left(C_{D}(\lambda)\right) \cdot \operatorname{det}\left(\operatorname{tridiag}\left(a_{1}(\lambda), b_{1}(\lambda), c_{1}(\lambda)\right)\right)
$$

where

$$
C_{D}(\lambda)=\left[\begin{array}{ll}
\xi_{1}^{\prime}\left(\sigma_{1}\right)-\lambda \xi_{1}\left(\sigma_{1}\right) & \xi_{3}^{\prime}\left(\sigma_{1}\right)-\lambda \xi_{3}\left(\sigma_{1}\right) \\
\xi_{1}^{\prime}\left(\sigma_{2}\right)-\lambda \xi_{1}\left(\sigma_{2}\right) & \xi_{3}^{\prime}\left(\sigma_{2}\right)-\lambda \xi_{3}\left(\sigma_{2}\right)
\end{array}\right],
$$


and

$$
\begin{aligned}
& a_{1}(\lambda)=-\left(1-\sigma_{1}\right)\left(1-\sigma_{2}\right)\left(\sigma_{2}-\sigma_{1}\right) \times \\
& \times\left[\left(1-\sigma_{1}\right)\left(1-\sigma_{2}\right) h^{2} \lambda^{2}+2\left(2-\sigma_{1}-\sigma_{2}\right) h \lambda+6\right] \\
& b_{1}(\lambda)=\left(\sigma_{2}-\sigma_{1}\right)\left\{\left[2 \sigma_{1} \sigma_{2}\left(1-\sigma_{1}\right)\left(1-\sigma_{2}\right)+\sigma_{1}\left(1-\sigma_{1}\right)+\sigma_{2}\left(1-\sigma_{2}\right)\right] h^{2} \lambda^{2}\right. \\
& -2\left(1-\sigma_{1}-\sigma_{2}\right)\left(1+\sigma_{1}+\sigma_{2}-2 \sigma_{1} \sigma_{2}\right) h \lambda \\
& \left.+6\left(1+2 \sigma_{1} \sigma_{2}-\sigma_{1}-\sigma_{2}\right)\right\} \\
& c_{1}(\lambda)=-\sigma_{1} \sigma_{2}\left(\sigma_{2}-\sigma_{1}\right)\left(\sigma_{1} \sigma_{2} h^{2} \lambda^{2}-2\left(\sigma_{1}+\sigma_{2}\right) h \lambda+6\right) \text {. }
\end{aligned}
$$

By noting some simple properties of tridiagonal Toeplitz matrices, (14) can be rewritten by

$$
\operatorname{det}\left(A_{1}-\lambda A_{0}\right)=h^{-2 N} \operatorname{det}\left(C_{D}(\lambda)\right) \cdot \prod_{j=1}^{N-1}\left(b_{1}(\lambda)+2 \sqrt{a_{1}(\lambda) c_{1}(\lambda)} \cos \frac{j \pi}{N}\right) .
$$

Consider the two special eigenvalues which satisfy the following quadratic equation

$$
\operatorname{det}\left(C_{D}(\lambda)\right)=\left(\sigma_{1}-\sigma_{2}\right)\left(\gamma_{0}+\gamma_{1} h \lambda+\gamma_{2} h^{2} \lambda^{2}\right)=0
$$

where

$$
\begin{aligned}
& \gamma_{0}=6 \sigma_{1} \sigma_{2}+2-3 \sigma_{1}-3 \sigma_{2} \\
& \gamma_{1}=\left(1-\sigma_{1}-\sigma_{2}\right)\left(2 \sigma_{1} \sigma_{2}-\sigma_{1}-\sigma_{2}\right) \\
& \gamma_{2}=\sigma_{1} \sigma_{2}\left(1-\sigma_{1}\right)\left(1-\sigma_{2}\right)>0 .
\end{aligned}
$$


Let $p_{D}\left(\sigma_{1}, \sigma_{2}\right):=\gamma_{1}^{2}-4 \gamma_{0} \gamma_{2}$. Then by $(19)$

$$
\begin{aligned}
& p_{D}\left(\sigma_{1}, \sigma_{2}\right)=\left(1+2 \sigma_{1} \sigma_{2}-\sigma_{1}-\sigma_{2}\right) \times \\
& \times\left[2 \sigma_{1}^{3} \sigma_{2}+2 \sigma_{1} \sigma_{2}^{3}-8 \sigma_{1}^{2} \sigma_{2}^{2}-\sigma_{1}^{3}-\sigma_{2}^{3}+5 \sigma_{1}^{2} \sigma_{2}+5 \sigma_{1} \sigma_{2}^{2}-6 \sigma_{1} \sigma_{2}+\sigma_{1}^{2}+\sigma_{2}^{2}\right]
\end{aligned}
$$

If $p_{D}\left(\sigma_{1}, \sigma_{2}\right)<0$, (18) has two complex roots and the real parts of these two roots are positive when $\gamma_{0}<0$. For those collocation points satisfying $p_{D}\left(\sigma_{1}, \sigma_{2}\right) \geq 0$, (18) has two positive real roots (or two negative real roots) when $\gamma_{0}>0$ and one positive and one negative roots when $\gamma_{0}<0$. Since $p_{D}\left(\sigma_{1}, \sigma_{2}\right)>0$ when $\gamma_{0}<0$, we let

$$
U^{D}=\left\{\left(\sigma_{1}, \sigma_{2}\right) \mid \gamma_{0}<0\right\}
$$

denote the unstable collocation point set. A plot of the boundaries of these collocation point sets is given in Figure 1. It follows that $U^{D}$ is an unstable collocation point set just by considering the two special eigenvalues. Then $U^{D}$ is also an unstable region for (12). The other eigenvalues are determined by

$$
\prod_{j=1}^{N-1}\left(b_{1}(\lambda)+2 \sqrt{a_{1}(\lambda) c_{1}(\lambda)} \cos \frac{j \pi}{N}\right)=0 .
$$

The stable collocation point sets for the eigenvalue problem (12) are described in the following theorem. The proof can be obtained by using Maple software for $(22)$. 


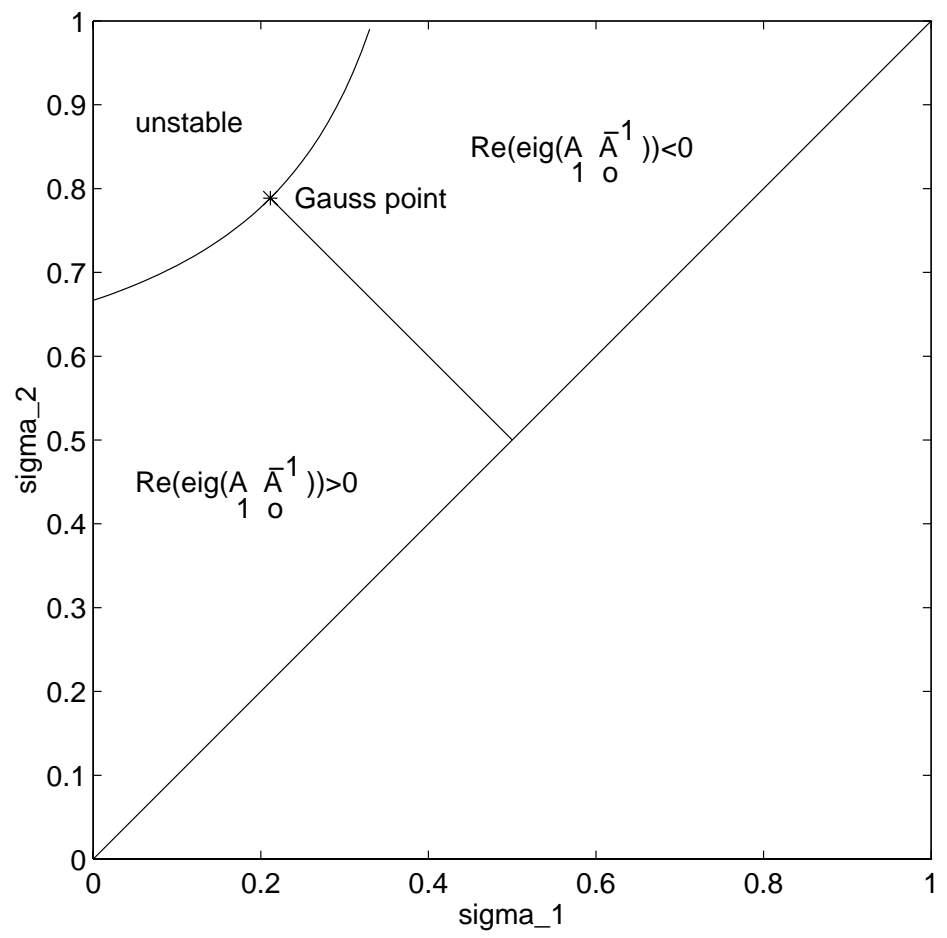

Figure 1: The collocation point sets for Dirichlet problems. 
Theorem 2 The stable collocation point sets are given by

$$
\begin{aligned}
& S^{D^{+}}=\left\{\left(\sigma_{1}, \sigma_{2}\right) \mid \gamma_{0}<0 ; \sigma_{1}+\sigma_{2}>1\right\} \\
& S^{D^{-}}=\left\{\left(\sigma_{1}, \sigma_{2}\right) \mid \gamma_{0}<0 ; \sigma_{1}+\sigma_{2}<1\right\} .
\end{aligned}
$$

Remarks: Our theoretical analysis in Theorem 2 confirms the numerical tests in [8]. In fact, the unstable and stable regions depend upon the two special eigenvalues which satisfy (18). It should be noted that $C_{D}(\lambda)$ is the characteristic polynomial of (12) when $N=1$, i.e., one element case. Thus the unstable region is independent of the step size $h$. Applying our analysis both to the convection-dispersion transport equations and the perturbation problems with $\epsilon$ small enough, the schemes with collocation points in $S^{D^{+}}$ (or $S^{D^{-}}$) are stable in $S^{D^{+}}$(or $S^{D^{-}}$) and unstable in $U^{D}$. In the latter case, there exist two eigenvalues with positive and negative real parts, respectively. The size of their real parts is $O(1 / h)$.

When $\epsilon \ll h$, the stability of (11) depends upon the ratio of the real part and imaginary part of the eigenvalues of (12). We define

$$
r\left(\sigma_{1}, \sigma_{2}\right)=\min \frac{\Re\left(\lambda_{j}\left(A_{1} A_{0}^{-1}\right)\right)}{\Im\left(\lambda_{j}\left(A_{1} A_{0}^{-1}\right)\right)} .
$$

It is difficult to find the optimal collocation point in the stability viewpoint. We have plotted the contours of $r\left(\sigma_{1}, \sigma_{2}\right)$ in Figure 2. Some features can be observed. When collocation points are chosen near by the symmetric case $\left(\sigma_{1}+\sigma_{2}=1\right)$ and the case $\sigma_{1}=\sigma_{2}$, the scheme is less stable. In the area near the point $(0,0.125), r\left(\sigma_{1}, \sigma_{2}\right)$ has its maximum which is larger than 2 . 


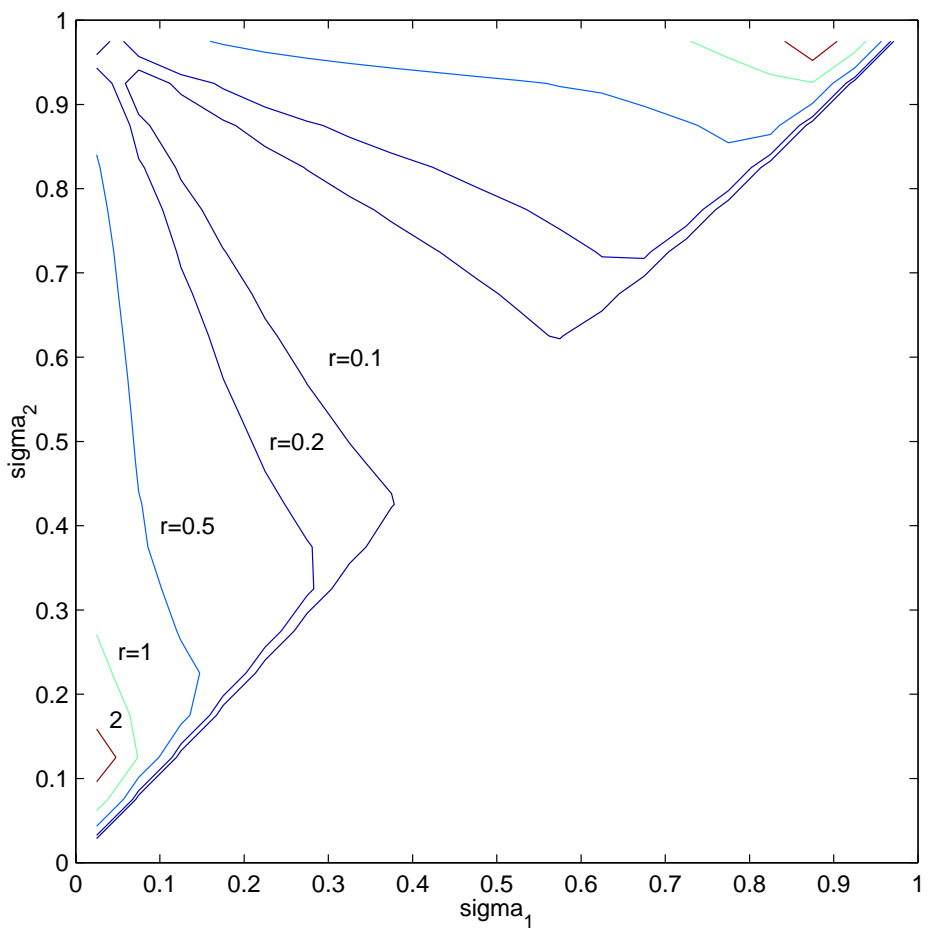

Figure 2: The collocation point sets for Dirichlet problems. 


\section{$4 \quad$ Numerical examples}

Finally, we present the results of calculations for the perturbed problems without turning points considered in [4]. We only test the stability to confirm our theoretical analysis for the Hermite cubic collocation method. In all examples, the error is measured by

$$
\|e\|_{\infty}=\max _{i}\left|v\left(x_{j}\right)-u\left(x_{j}\right)\right|
$$

where $v(x)$ and $u(x)$ denote the numerical solution and the analytic solution (if available). All numerical experiments are performed on a Sun SPARC Station $1+$ in double precision.

\subsection{Example 1}

We consider the singular perturbation problem

$$
\begin{aligned}
& 4 \epsilon u_{x x}-2 u_{x}=\frac{1+x}{2} \quad x \in(-1,1) \\
& u(-1)=u(1)=0
\end{aligned}
$$

which was studied by Huang and Sloan [4] by using a so-called upwinding pseudospectral collocation methods. It has been shown in [4] that the standard pseudospectral collocation method produces a bad oscillation when $\epsilon<10^{-3}$. Here we use the Hermite cubic spline collocation method for solving this perturbation problem. Numerical results are given in Table 1 with 
several different collocation points, including the Gauss points. It is obvious that when $\epsilon \leq 10^{-3}$, the numerical solutions obtained by the collocation method using Gauss points are unacceptable. We plot the numerical results obtained by the upwinding collocation method based on Hermite cubic spline and the corresponding orthogonal collocation method in Figure 3 and Figure 4, respectively. The numerical results are improved significantly by using the nonsymmetric collocation points with upwinding features. The collocation method with the collocation point $(0.875,0.95)$ gives the best results. The accuracy is independent of the value of $\epsilon$. These numerical observations are in good agreement with our theoretical analysis.

TABLE 1: The numerical comparison for Example $4.1(N=16)$

\begin{tabular}{|c|c|c|c|c|c|}
\hline $\begin{array}{c}\text { Collocation point } \\
\left(\sigma_{1}, \sigma_{2}\right)\end{array}$ & $\begin{array}{c}\|e\|_{\infty} \\
\epsilon=10^{-1}\end{array}$ & $\begin{array}{c}\|e\|_{\infty} \\
\epsilon=10^{-2}\end{array}$ & $\begin{array}{c}\|e\|_{\infty} \\
\epsilon=10^{-3}\end{array}$ & $\begin{array}{c}\|e\|_{\infty} \\
\epsilon=10^{-4}\end{array}$ & $\begin{array}{c}\|e\|_{\infty} \\
\epsilon=10^{-5}\end{array}$ \\
\hline $\begin{array}{c}\text { Gauss point } \\
(0.2,0.8) \\
(\text { unstable })\end{array}$ & $1.615 \mathrm{D}-5$ & $0.7409 \mathrm{D}-2$ & $0.4075 \mathrm{D} 0$ & $0.4639 \mathrm{D} 0$ & $0.4683 \mathrm{D} 0$ \\
\hline$(0.6,0.9)$ & $5.656 \mathrm{D}-2$ & $2.873 \mathrm{D}-2$ & $3.613 \mathrm{D}-2$ & $3.694 \mathrm{D}-2$ & $3.711 \mathrm{D}-2$ \\
\hline$(0.875,0.95)$ & $4.765 \mathrm{D}-3$ & $1.1341 \mathrm{D}-2$ & $2.2963 \mathrm{D}-3$ & $3.581 \mathrm{D}-3$ & $3.741 \mathrm{D}-3$ \\
\hline $\begin{array}{c}\text { FD (upwind) } \\
(N=100)\end{array}$ & $9.774 \mathrm{D}-3$ & $6.719 \mathrm{D}-2$ & $4.983 \mathrm{D}-2$ & $9.850 \mathrm{D}-3$ & $5.444 \mathrm{D}-3$ \\
\hline $\begin{array}{c}\text { FD (central) } \\
(N=100)\end{array}$ & $1.227 \mathrm{D}-4$ & $1.693 \mathrm{D}-2$ & $0.3327 \mathrm{D} 0$ & $0.4986 \mathrm{D} 0$ & $2.527 \mathrm{D} 0$ \\
\hline
\end{tabular}



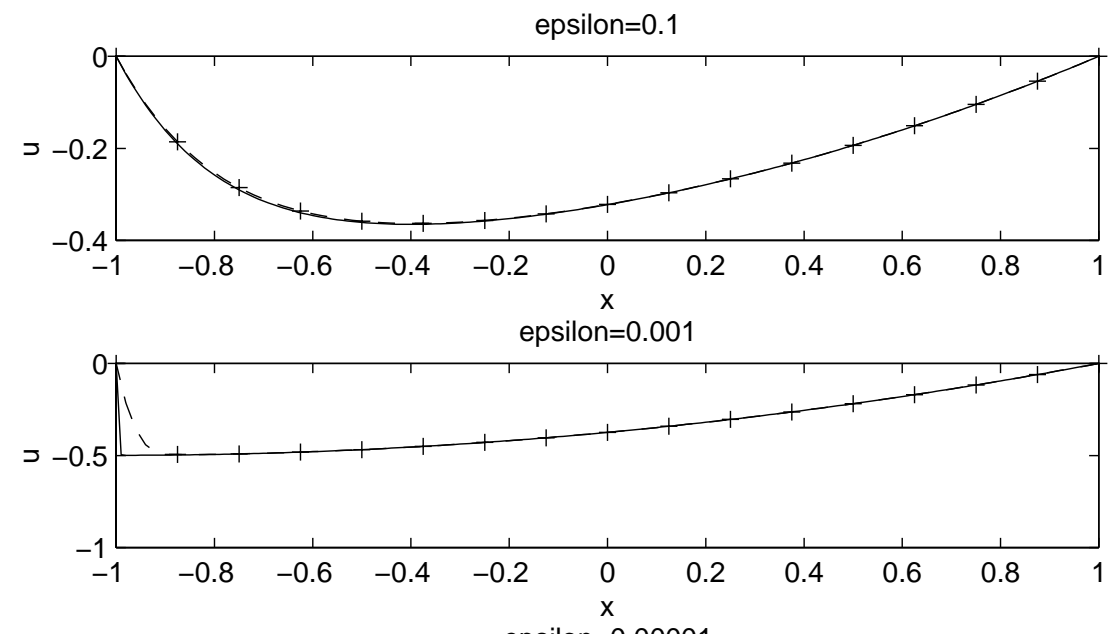

epsilon $=0.00001$

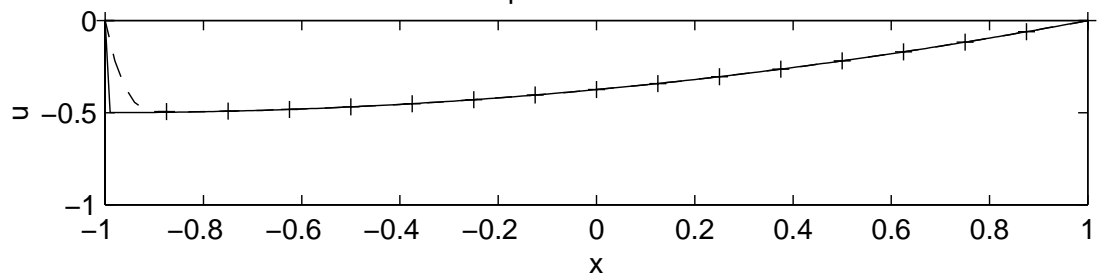

FiguRE 3: Numerical comparison for Example 4.1 (exact solution: -; upwinding collocation solution: + ). 


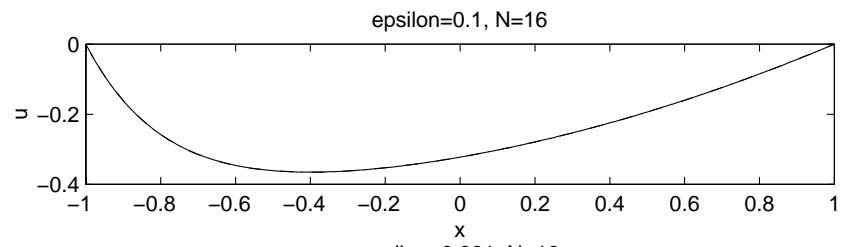

epsilon $=0.001, \mathrm{~N}=16$

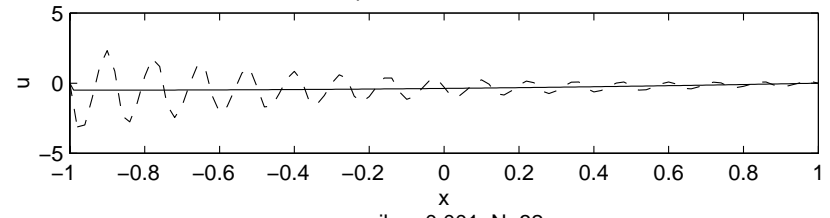

epsilon $=0.001, \mathrm{~N}=32$

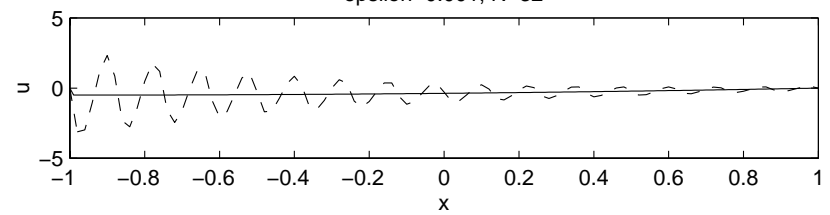

epsilon $=0.001, \mathrm{~N}=64$

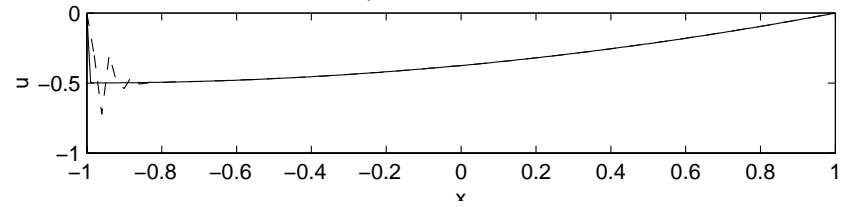

Figure 4: Numerical comparison for Example 4.1 (exact solution: -; orthogonal collocation solution: ---;). 


\subsection{Example 2}

The second example is a perturbation problem with variable coefficients [4]

$$
4 \epsilon u_{x x}+2 p(x) u_{x}=f(x, \epsilon) \quad x \in(-1,1)
$$

where $p(x)=(3 / 2+x / 2)^{3}$, and $f(x, \epsilon)$ and the boundary conditions are chosen such that the exact solution of $(27)$ is

$$
u(x)=\frac{1}{p(x)} \exp \left(-\frac{1}{2 \epsilon} \int_{-1}^{x} p(t) d t\right)+\exp \left(-\frac{x+1}{4}\right) .
$$

Numerical results are given in Table 2. These reinforce conclusions obtained for Example 4.1.

Acknowledgements: Dedicated to Professor M. R. Osborne on the occasion of his 65th birthday. The author would like to thank the referees for their many useful comments that result in the improvement of this presentation. This work was supported in part by Hong Kong RGC grant 9040467.

\section{References}

[1] M.B. Allen and G.F. Pinder. Collocation simulation of multiphase porous-medium flow. J. Society of Petroleum Engineers, Feb.:135-142, 1983. C1381, C1381 
TABLE 2: The numerical comparison for Example $4.2(N=16)$

\begin{tabular}{|c|c|c|c|c|c|}
\hline $\begin{array}{c}\text { Collocation point } \\
\left(\sigma_{1}, \sigma_{2}\right)\end{array}$ & $\begin{array}{c}\|e\|_{\infty} \\
\epsilon=10^{-1}\end{array}$ & $\begin{array}{c}\|e\|_{\infty} \\
\epsilon=10^{-2}\end{array}$ & $\begin{array}{c}\|e\|_{\infty} \\
\epsilon=10^{-3}\end{array}$ & $\begin{array}{c}\|e\|_{\infty} \\
\epsilon=10^{-4}\end{array}$ & $\begin{array}{c}\|e\|_{\infty} \\
\epsilon=10^{-5}\end{array}$ \\
\hline Gauss point & $1.234 \mathrm{D}-4$ & $0.1482 \mathrm{D}-1$ & $0.6877 \mathrm{D} 0$ & $0.7967 \mathrm{D} 0$ & $0.8087 \mathrm{D} 0$ \\
\hline $\begin{array}{c}(0.2,0.8) \\
(\text { unstable })\end{array}$ & $3.413 \mathrm{D}-4$ & $0.1256 \mathrm{D} 0$ & 0.0 .8909 & 0.9333 & 0.9371 \\
\hline$(0.6,0.9)$ & $5.882 \mathrm{D}-3$ & $1.054 \mathrm{D}-2$ & $5.961 \mathrm{D}-2$ & $7.252 \mathrm{D}-2$ & $7.392 \mathrm{D}-2$ \\
\hline$(0.875,0.95)$ & $1.150 \mathrm{D}-2$ & $1.603 \mathrm{~d}-2$ & $4.950 \mathrm{D}-3$ & $7.216 \mathrm{D}-3$ & $7.488 \mathrm{D}-3$ \\
\hline $\begin{array}{c}\text { FD (upwind) } \\
(N=100)\end{array}$ & $2.392 \mathrm{D}-2$ & 0.1384 & $9.737 \mathrm{D}-2$ & $1.966 \mathrm{D}-2$ & $1.112 \mathrm{D}-2$ \\
\hline $\begin{array}{c}\text { FD (central) } \\
(N=100)\end{array}$ & $2.969 \mathrm{D}-3$ & $1.339 \mathrm{D}-2$ & $0.6077 \mathrm{D} 0$ & $1.410 \mathrm{D} 0$ & $12.414 \mathrm{D} 0$ \\
\hline
\end{tabular}

[2] U. M. Ascher, R. M. Mattheij, and R. D. Russell. Numerical Solution of Boundary Value Problems for Ordinary Differential Equations. Prentice-Hall, New Jersey, 1988. C1380, C1385

[3] G. Fairweather and D. Meade, A survey of spline collocation methods for the numerical solution of differential equations, In J.C. Diaz, editor, Mathematics for Large Scale Computing, Lecture Notes in Pure Appl.Math. 120, Marcel Dekker, New York, pages 297-340, 1989. C1380

[4] W. Huang and D. Sloan. A new pseudospectral method with upwind features. IMA J. Numer. Anal., 13:413-430, 1993. C1381, C1391, C1391, C1391, C1395 
[5] D. Mahmood and M.R. Osborne. Collocation for BVP's: compact and noncompact schemes. In D. Stewart, H. Gardner and D. Singleton, editors, Computational Techniques and Applications: CTAC93, pages 354-361, World Scientific, 1994. C1381, C1385

[6] C. Ringhofer. On collocation schemes for quasilinear singularly perturbed boundary value problems. SIAM J. Numer. Anal., 21:864-882, 1984. C1381, C1381

[7] R.D. Russell and W. Sun. Spline collocation differentiation matrices. SIAM J. Numer. Anal., 34:2274-2287, 1997. C1380

[8] W. Sun. The spectral analysis of Hermite cubic spline collocation systems. SIAM J. Numer. Anal., 36:1962-1975, 1999. C1382, C1385, C1385, C1385, C1389

[9] W. Sun. Block iterative algorithm for solving Hermite bicubic collocation equations. SIAM J. Numer. Anal., 33:589-601, 1996. C1385

[10] W. Sun. Fast algorithms for solving high-order spline collocation systems. Numer. Math., 81:143-160, 1998. C1385 